\title{
Manufacturing and optical performance of silicon immersed gratings for Sentinel-5
}

Ralf Kohlhaas

ICSO 2018

10-10-2018

\section{SRON}




\section{Introduction}

SRON supports green house gas observation missions with the delivery of immersed gratings

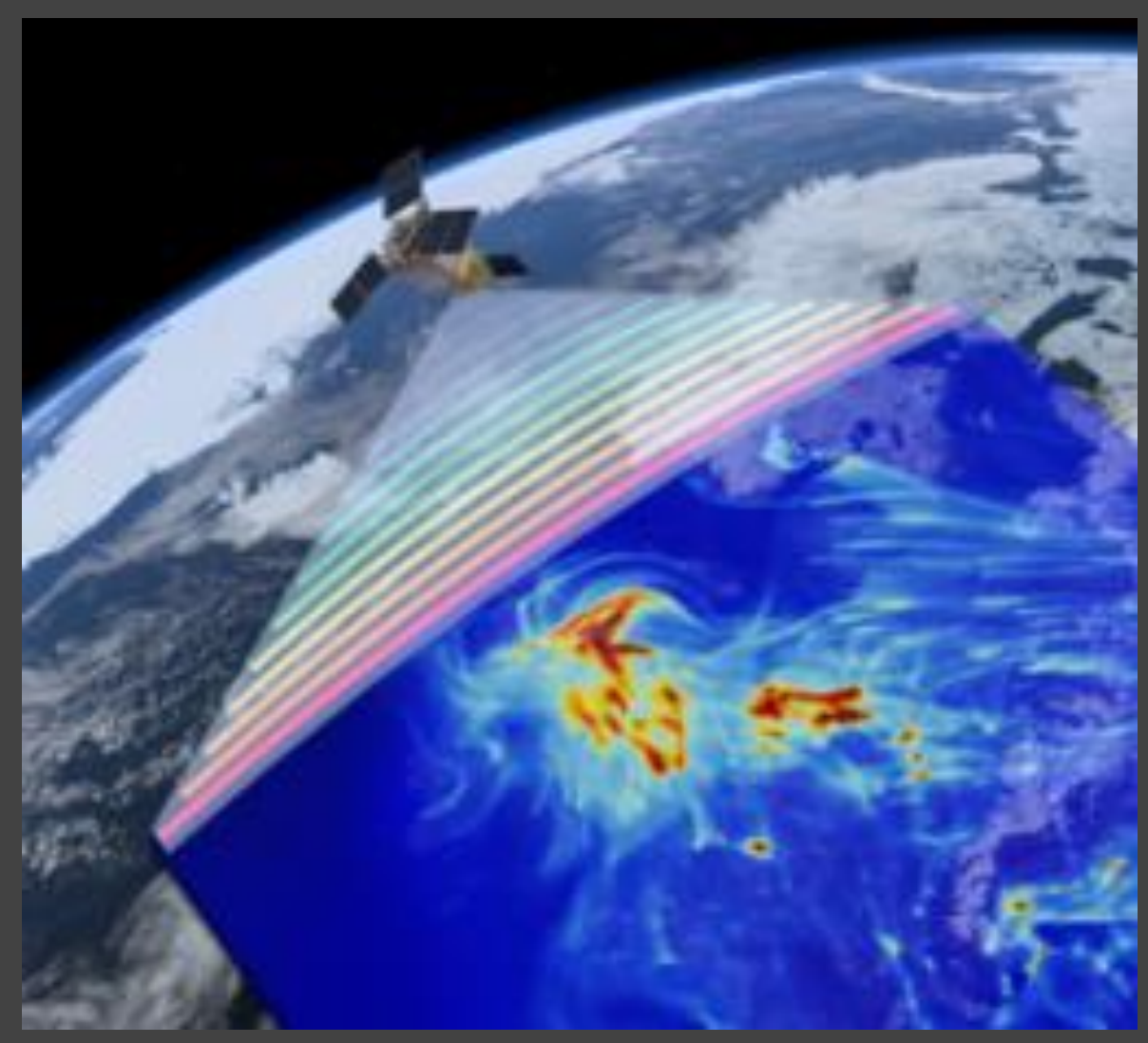

Recent example:

Immersed grating for Sentinel-5 precursor TROPOMI

Current challenge:

6 Flight models + spares for Sentinel-5

Image source: ESA 


\section{Outline}

1. Overview of Immersed Gratings for Sentinel-5

2. Immersed Grating production

3. Optical performance

3.1. Stray light

3.2. Wavefront error

3.3. Polarized efficiency

4. Environmental tests

5. Conclusions 


\section{Overview of Immersed Gratings for Sentinel-5}

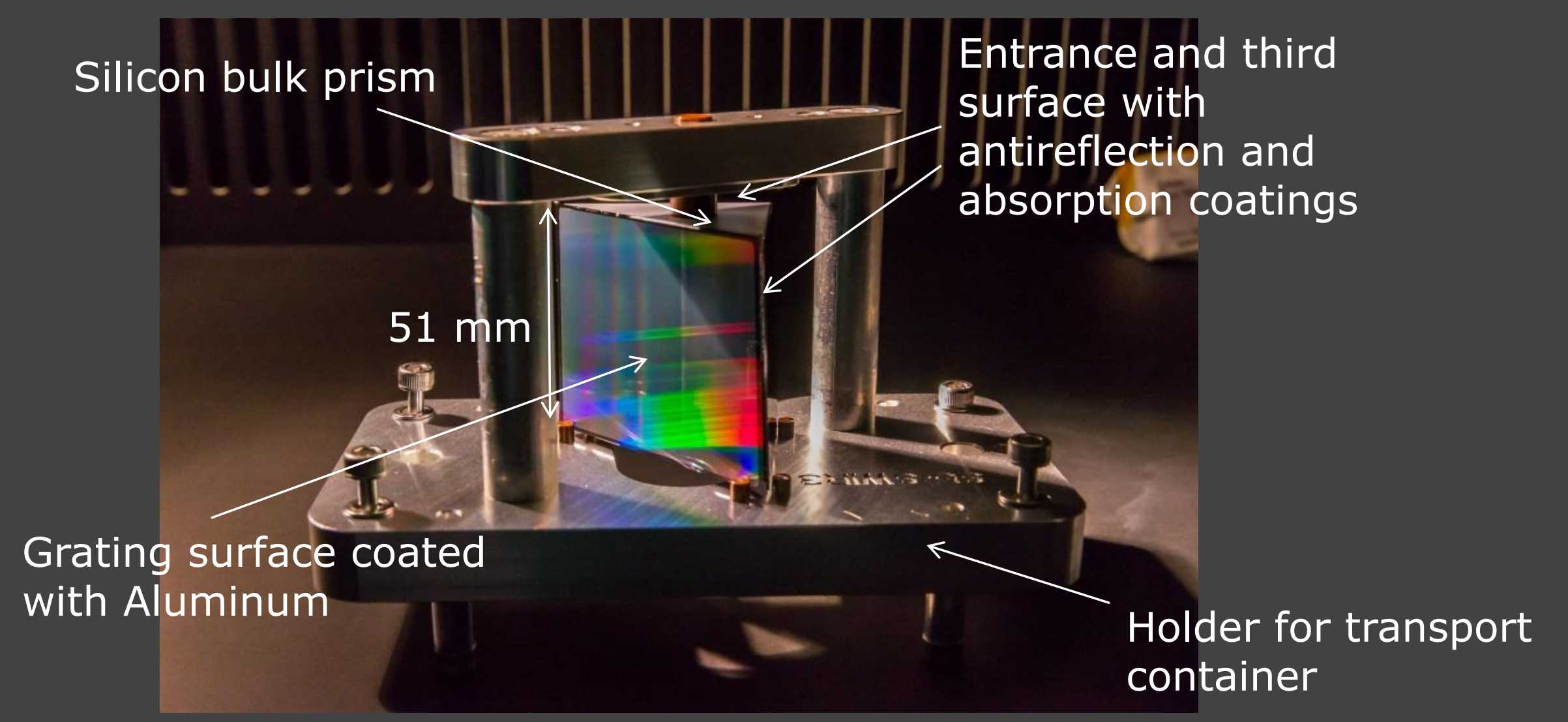

Light enters silicon and is reflected on grating surface

$\Rightarrow$ Increase in resolving power with factor $n=3.4$ ! 


\section{Overview of Immersed Gratings for Sentinel-5}

$\begin{array}{ll}\text { Two versions: } & \text { SWIR-1 }(1589 \mathrm{~nm}-1676 \mathrm{~nm}) \\ & \text { SWIR-3 }(2304 \mathrm{~nm}-2386 \mathrm{~nm})\end{array}$
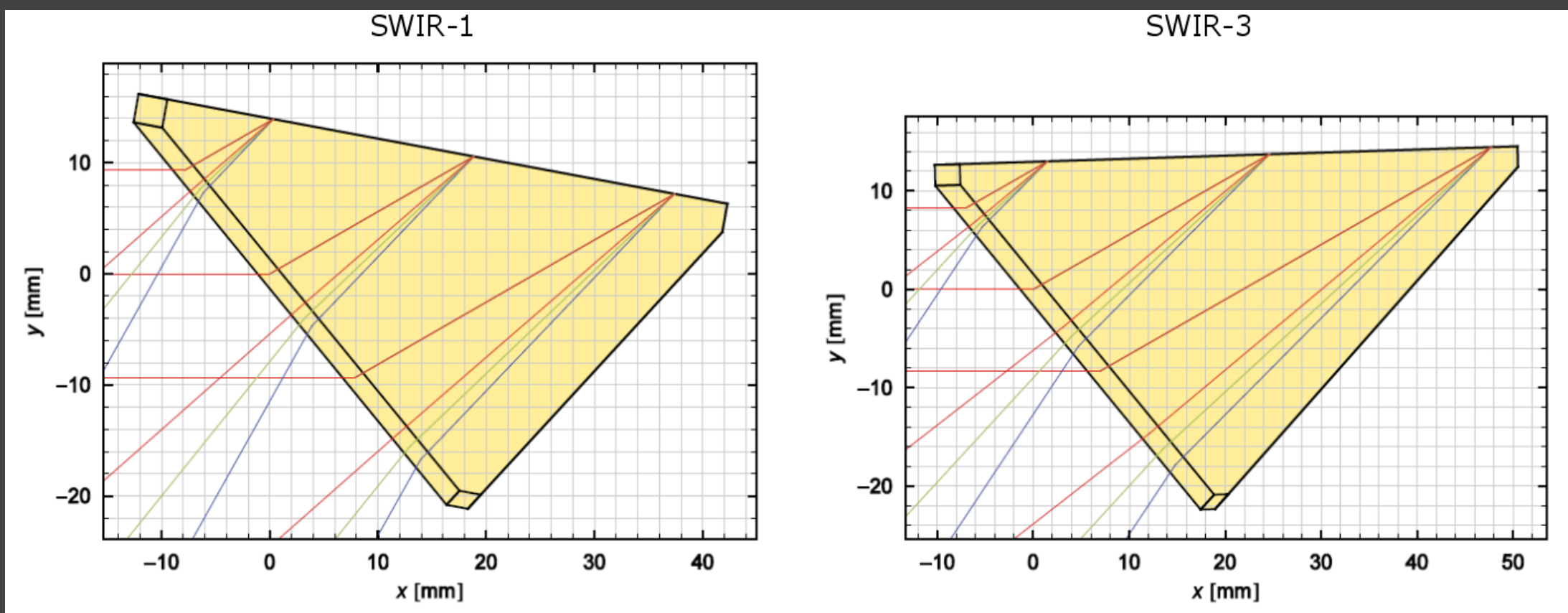

Grating design optimized to minimize internal reflections + entrance surface wedged s.t. reflections do not reach detector

\section{SRON}




\section{Immersed Grating production}

Direct bonding of a silicon grating element with a silicon prism.

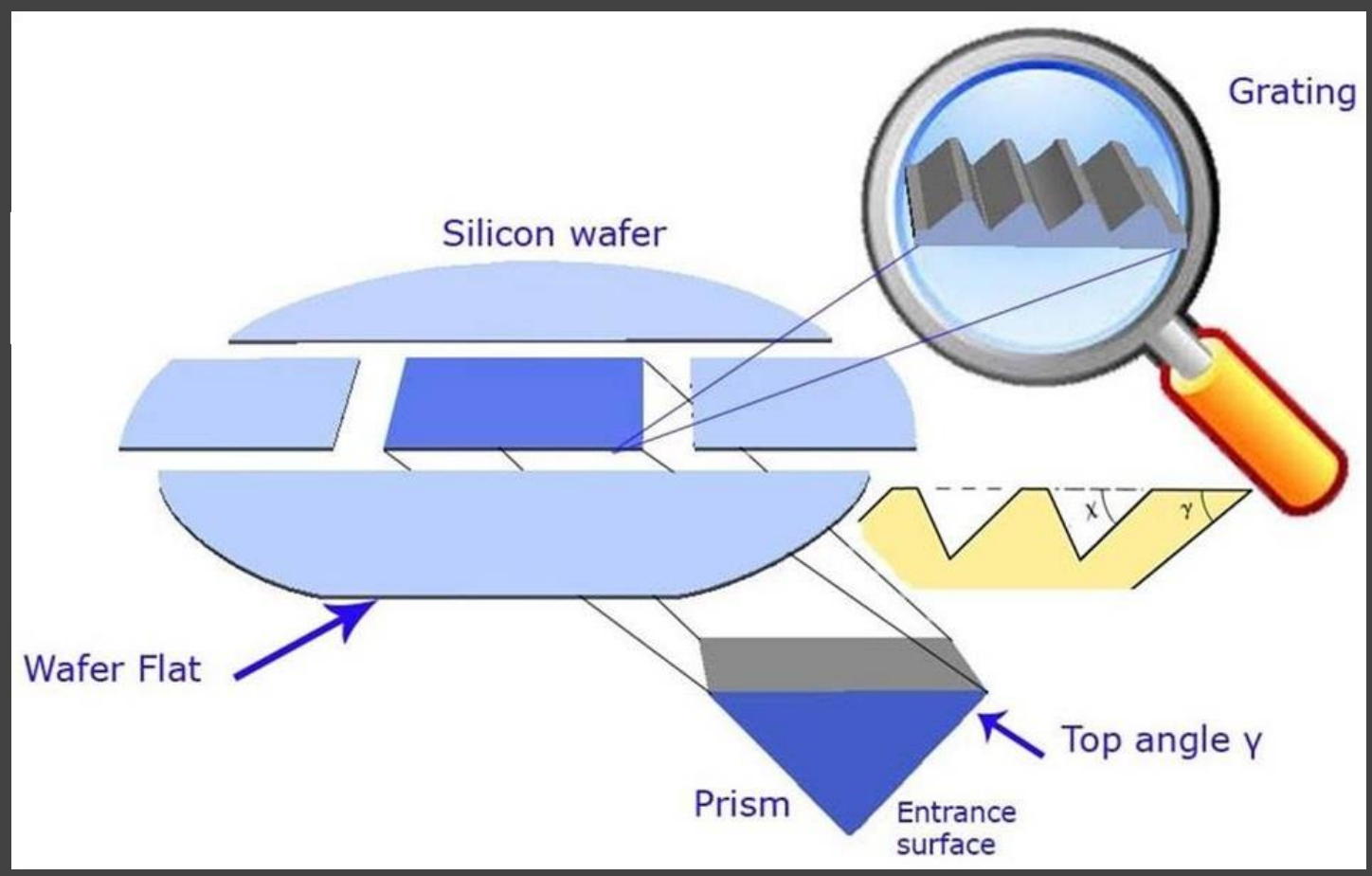

Steps:

1. Grating element production

2. Bond and fuse to prism

3. Scribe and break to remove excess wafer parts

4. Apply coatings

Production methods allows combination of best grating elements with prisms with tight angle tolerances 


\section{IG production - grating elements}

\section{Production method:}

- UV lithography

- anisotropic KOH etching for ultra-smooth surfaces

\section{Results:}

- Dam width $(380 \pm 15) \mathrm{nm}$

- Defect density $<1 \mathrm{e}-5$

- Grating surface roughness of $<0.5 \mathrm{~nm}$ rms

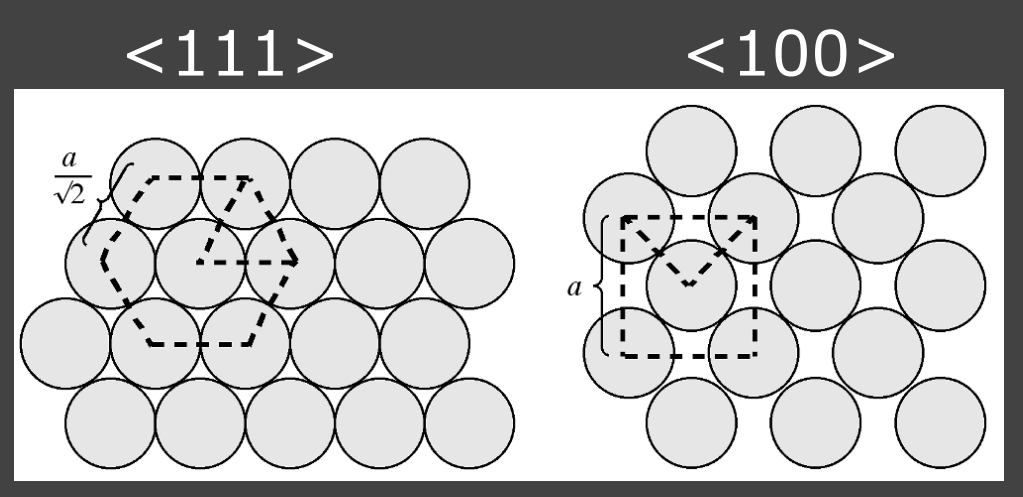

$\mathrm{KOH}$ two orders faster etching along $<100>$ than $<111>$

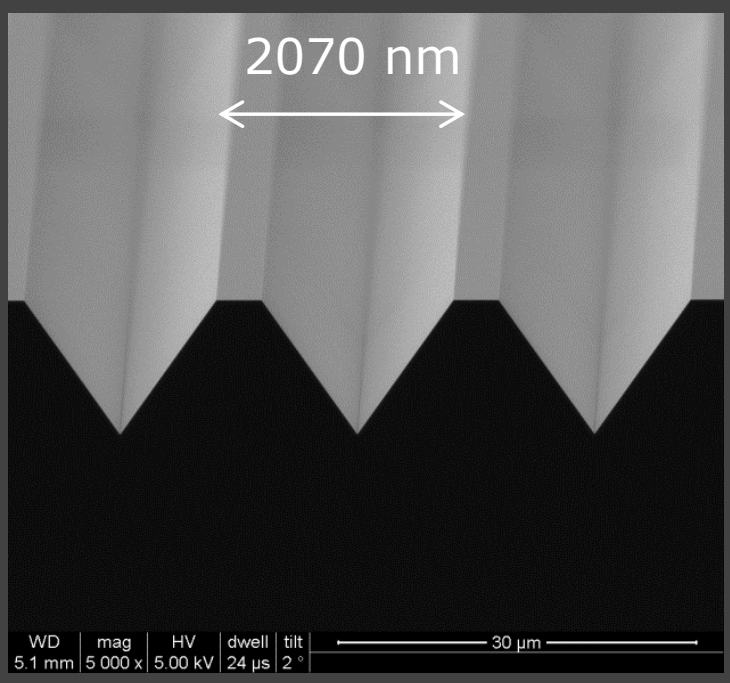

SEM image of etched grating element 


\section{IG production - bonding}

Principle: Direct contact bonding over atomic forces

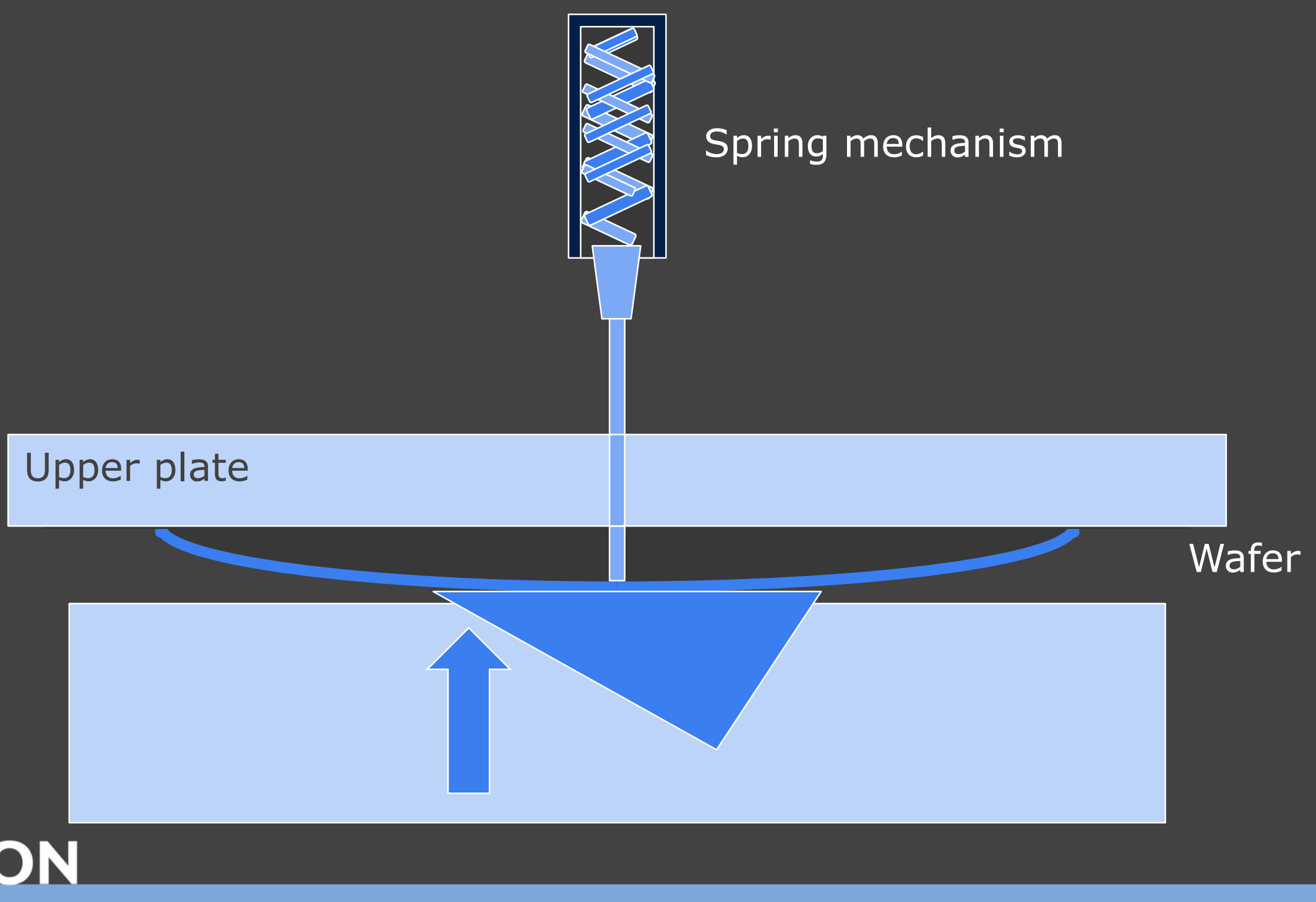




\section{IG production - bonding}

Wafer to wafer bonder modified for immersed grating bondings.

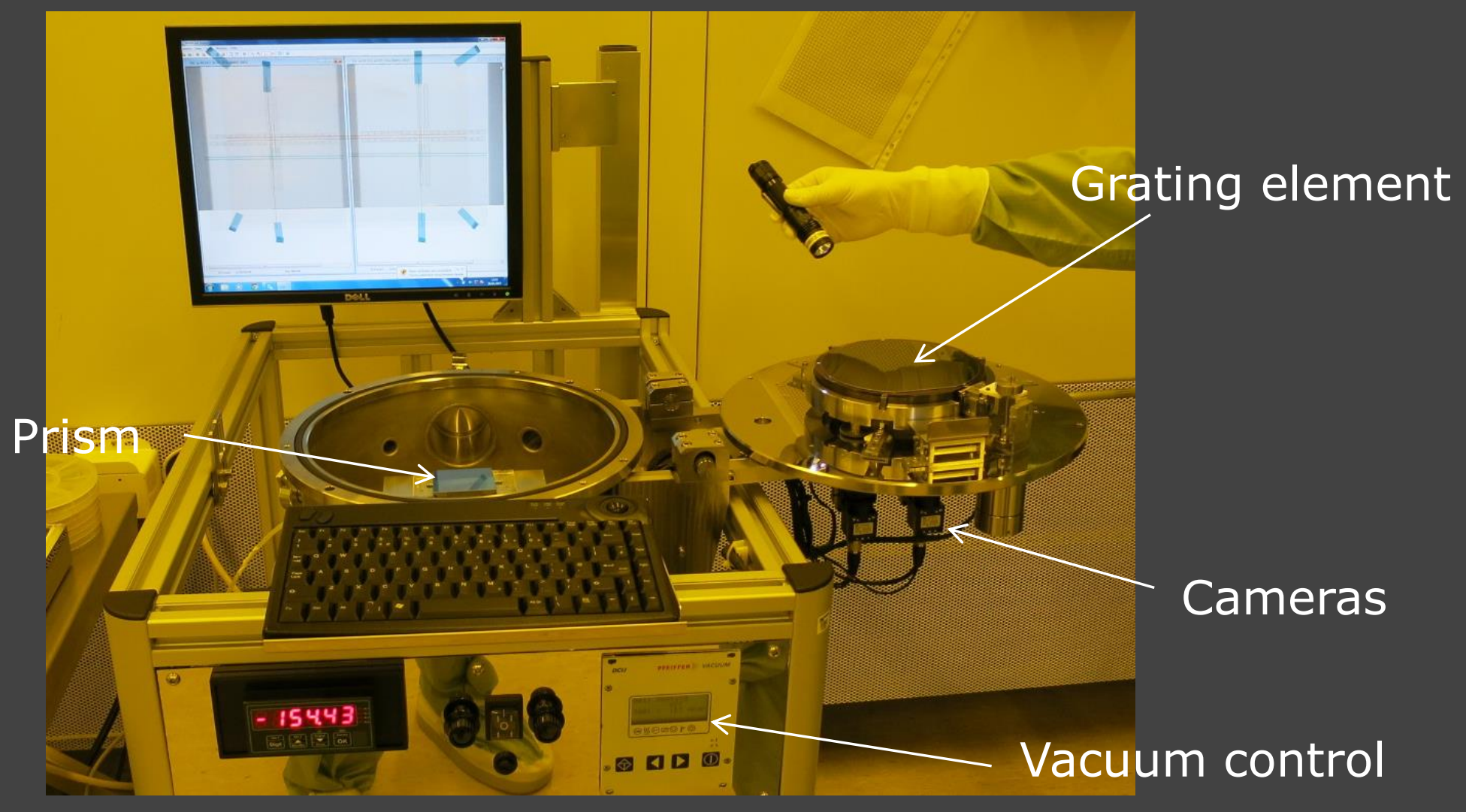

$\mathrm{CMM}+$ optical measurements for prism-wafer alignment. Rotation accuracy of $<100$ arcsec for all flight model bonds. 


\section{Optical performance - stray light}

For the first time challenging stray light measurements at 2.3 $\mu \mathrm{m}$ done at ESA-ESTEC (see also talk of D. Tomuta on Friday)

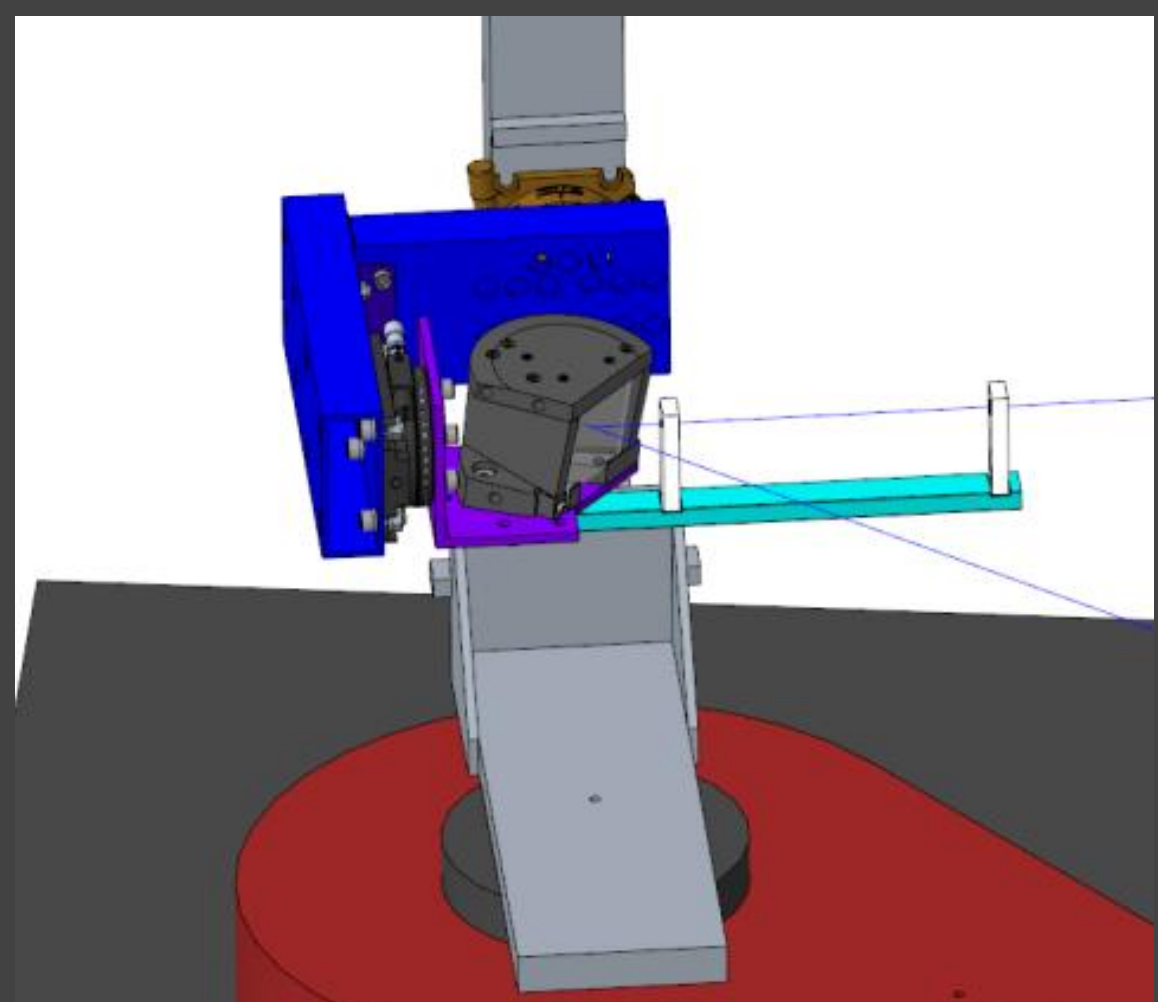

Pre-alignment done with mirror on platform

Optomechanics designed to allow simple replacement with immersed grating

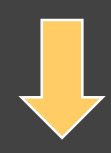

General approach applicable for complex components 


\section{Optical performance - stray light}

\section{Expected stray light distribution}

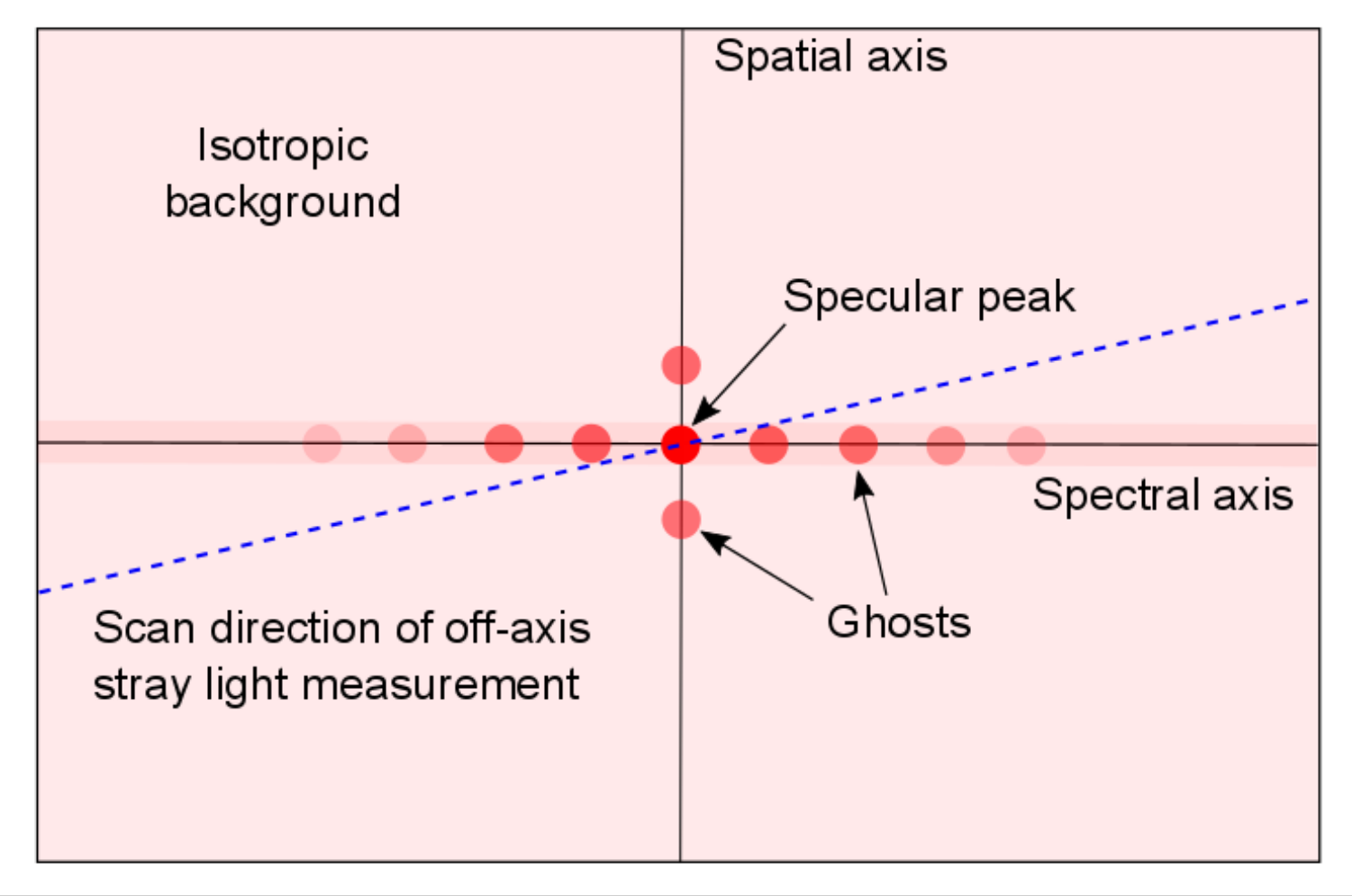

Ghosts from lithography mask writing errors

Radial symmetric background

$\Rightarrow$ Only line scans possible with setup 


\section{Optical performance - stray light}

Results of Bidirectional Reflectance distribution function (BRDF):

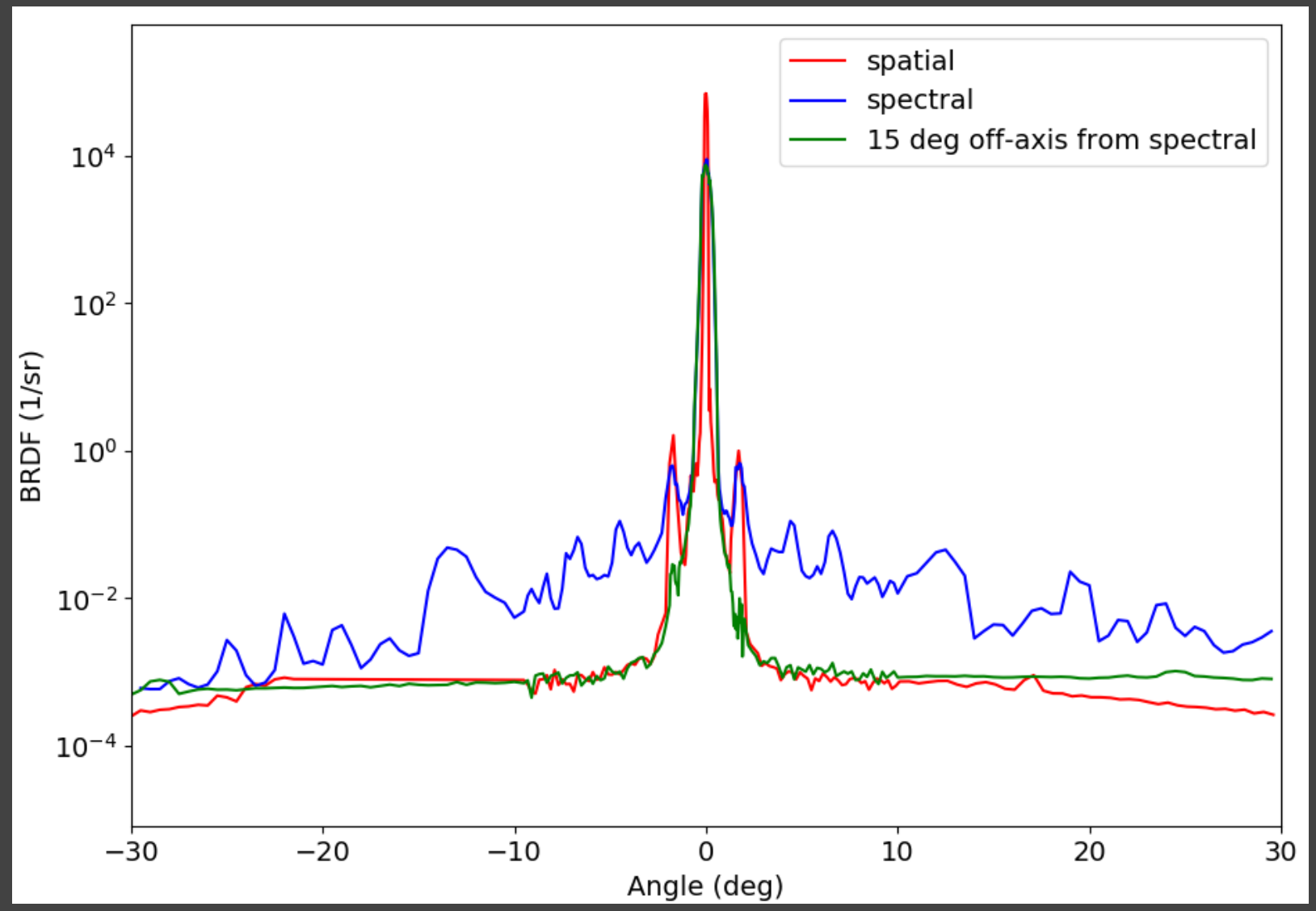

Background at $10^{-3}$ level

Total integrated scatter (TIS) excluding internal reflections $\approx 0.2 \%$

Relative ghost intensity $<10^{-4}$

Stray light the same for all 7 SWIR-3 IGs built 


\section{Optical performance - wavefront error}

Wavefront error measured from outside with Fizeau interferometer, result translated to operational conditions.

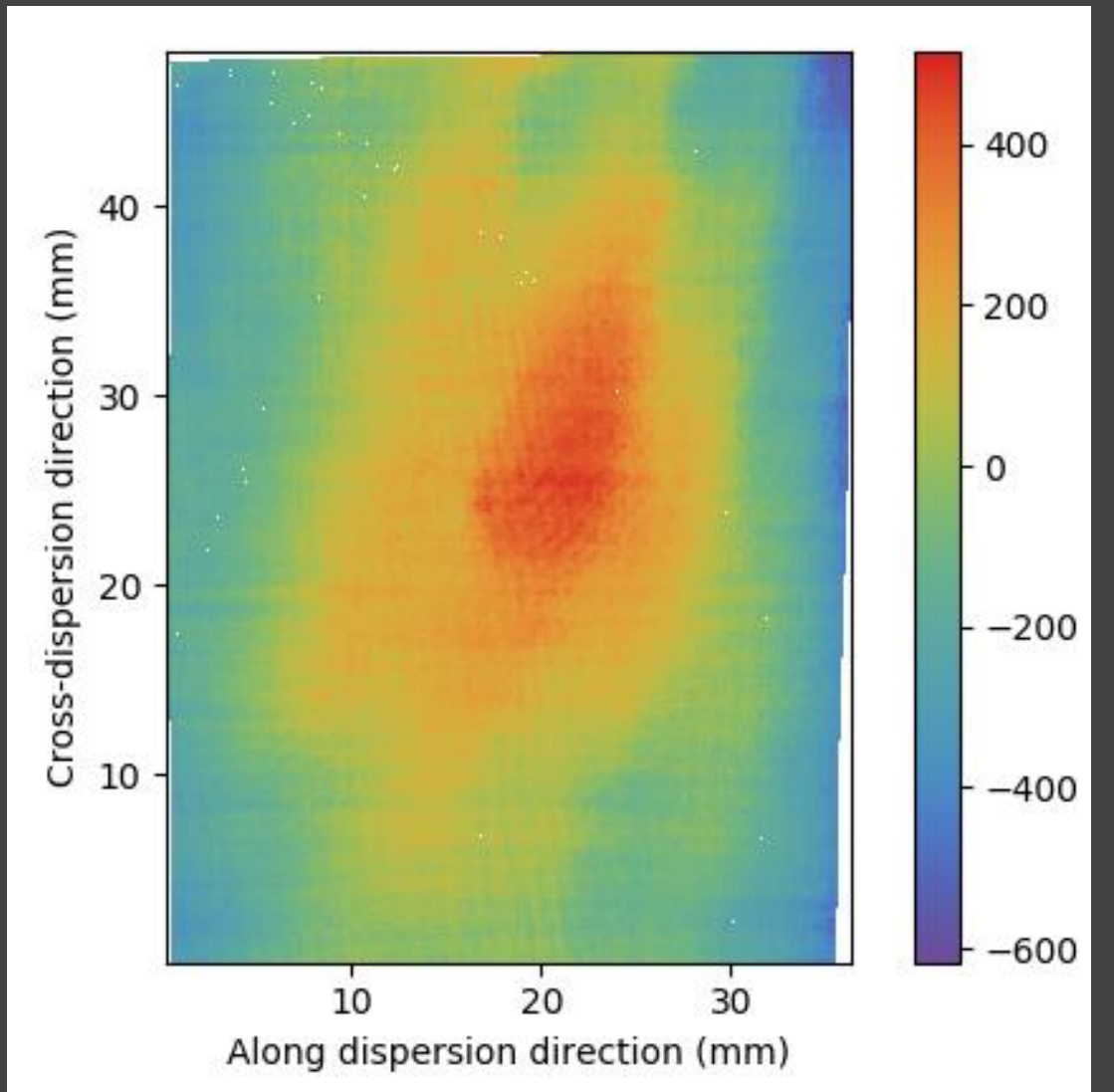

Results SWIR-3 IG (EM):

Total WFE $(206 \pm 20) \mathrm{nm}$ vs. requirement of $900 \mathrm{~nm}$

WFE with power removed of $(136 \pm 25) \mathrm{nm}$ vs requirement of $180 \mathrm{~nm}$

WFE dominated by wafer thickness variations 


\section{Optical performance - wavefront error}

Confirmation in immersion with experimental Shack-Hartmann setup, based on off-the-shelf components:

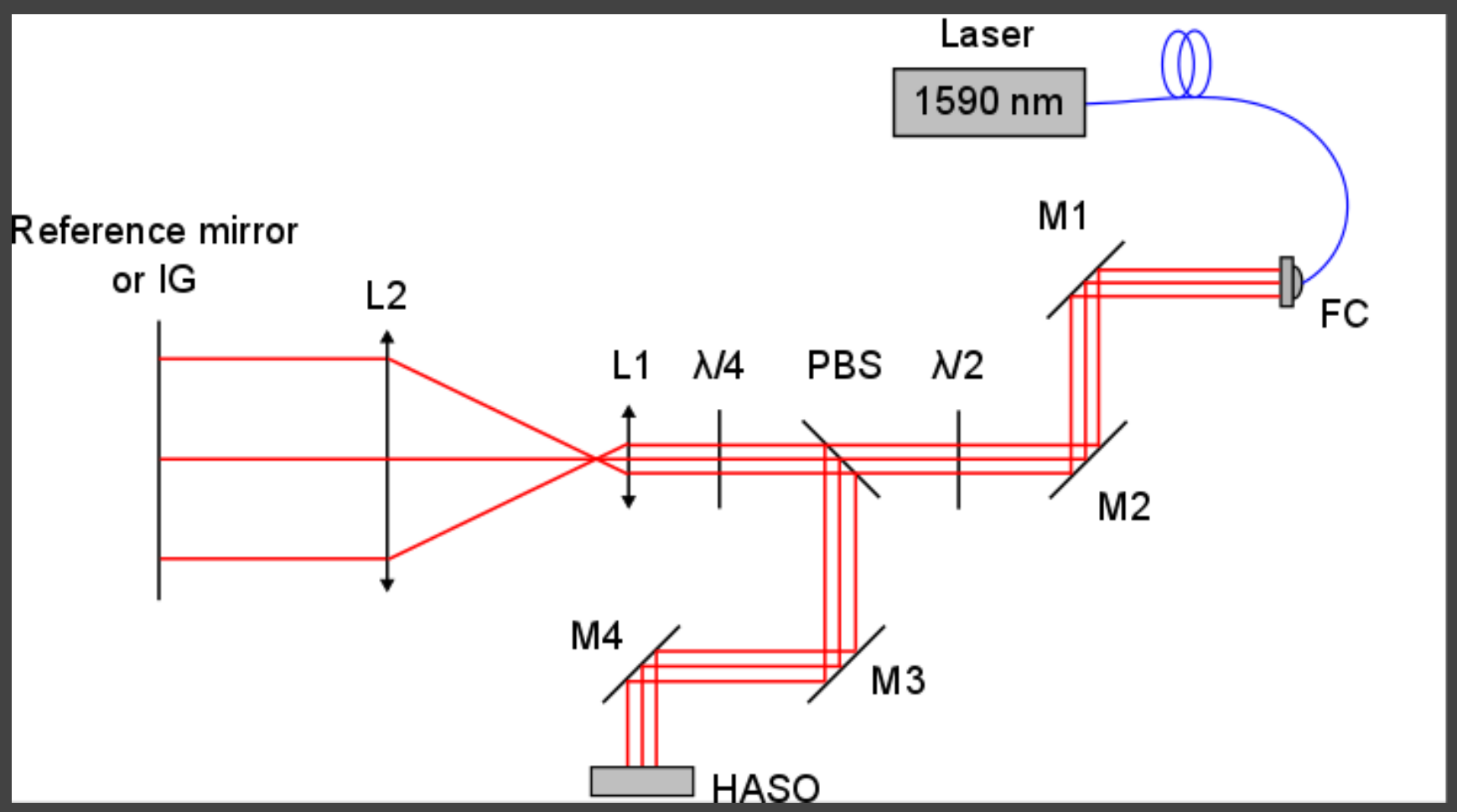

Results confirmed on limited diameter of $20 \mathrm{~mm}$ with accuracy of $-40 /+20 \mathrm{~nm}$. Setup could be improved with custom telescope. 


\section{Optical performance - polarized efficiency}

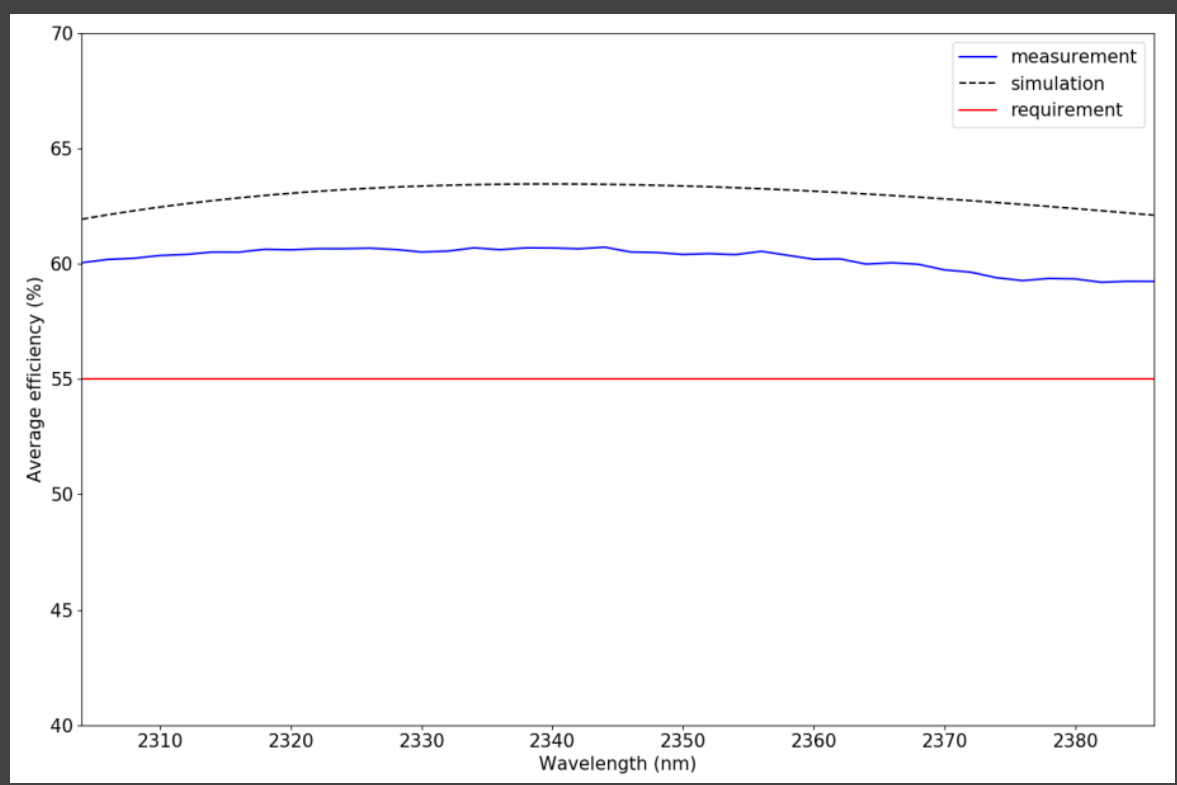

Average efficiency

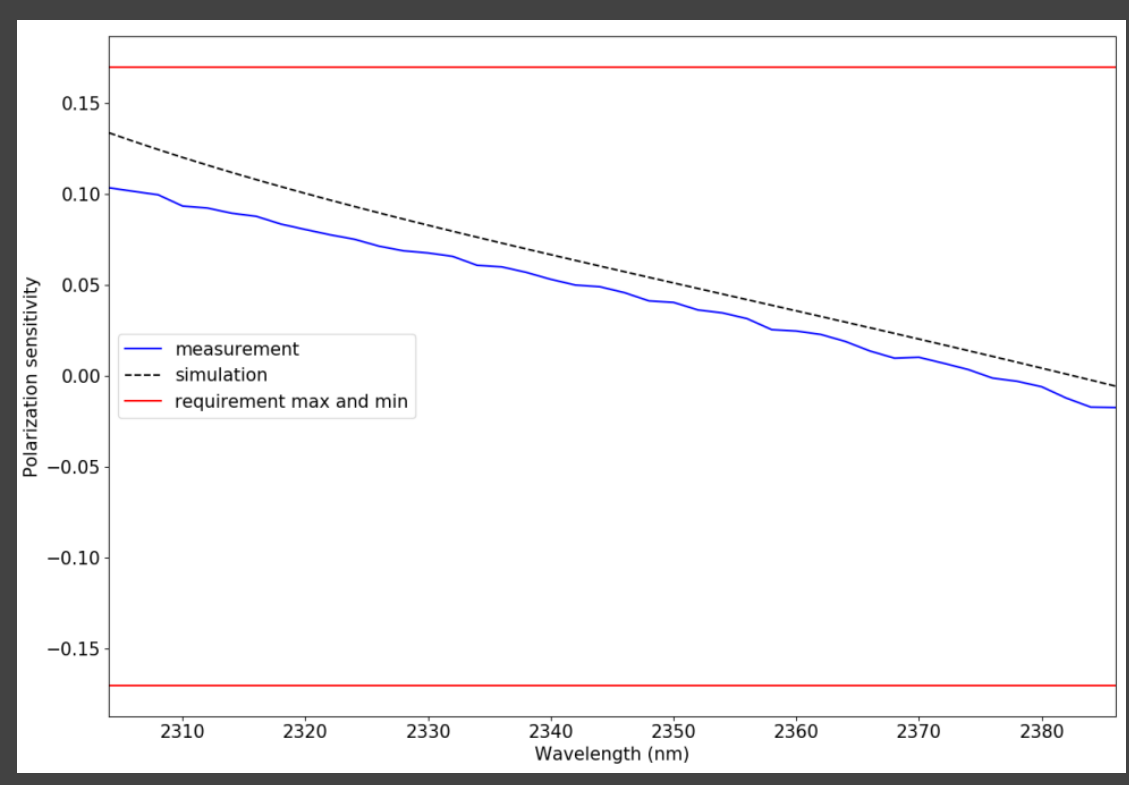

Polarization

Polarization sensitivity $(<0.12)$ and average efficiency $(>58 \%)$ close to simulation

Reason for lower efficiency than in simulation is unknown SRON 


\section{Environmental tests}

Immersed grating are delivered in mechanical flight housing and environmental tested

Dedicated TV setup for cryogenic performance tests and cycling (right)

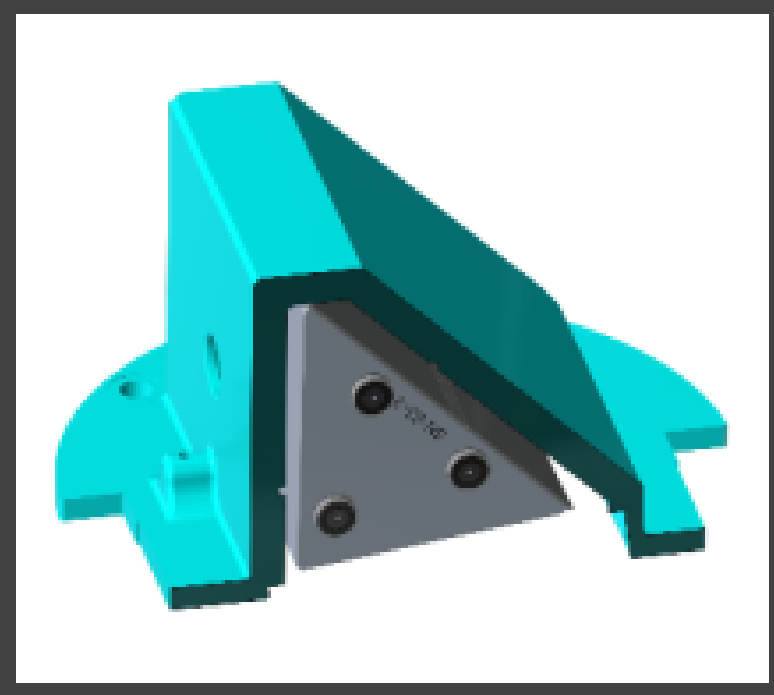

SRON

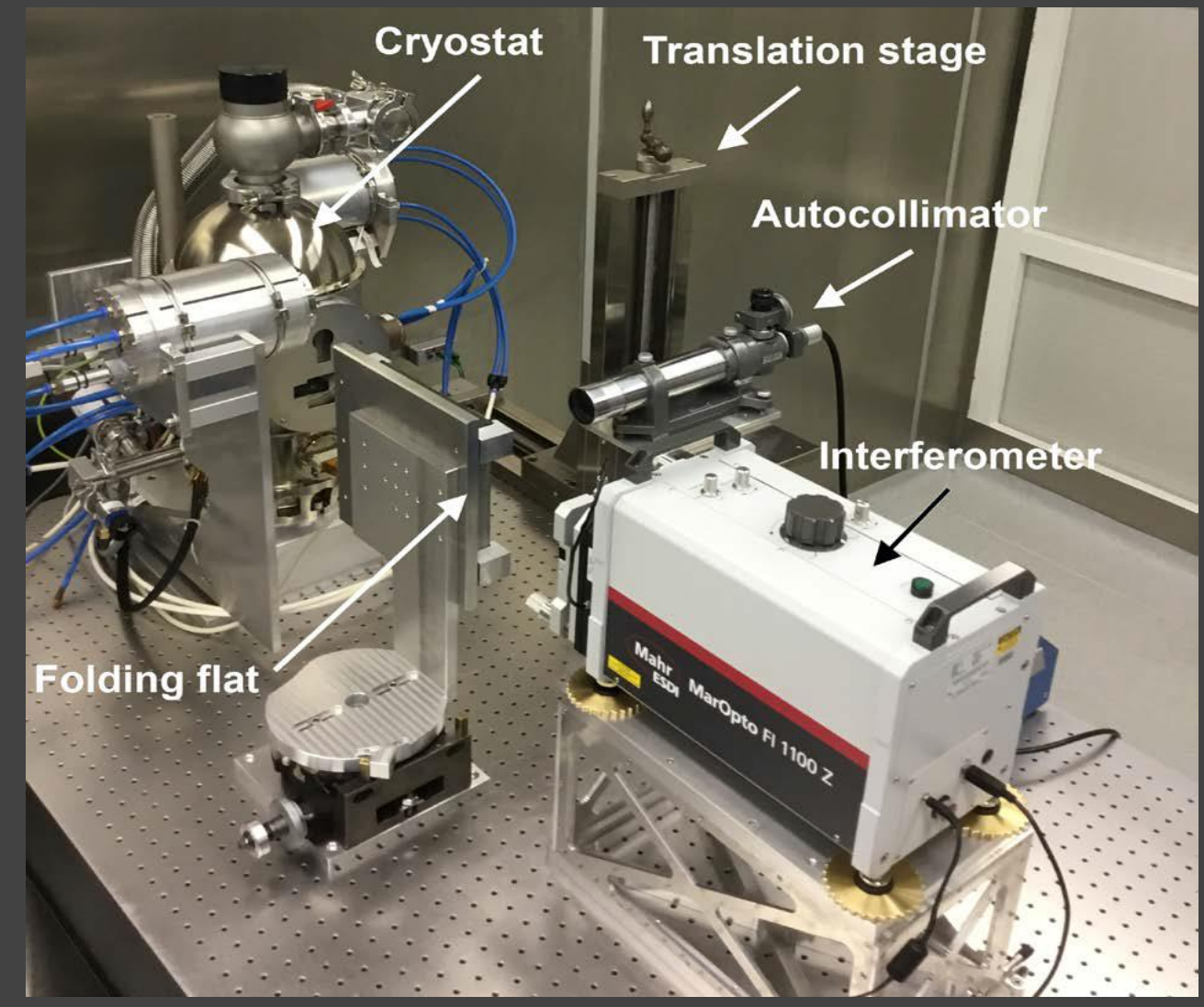

Qualification thermal vacuum and vibration tests were passed

$\Rightarrow$ Silicon bond qualified for space! 


\section{Conclusions}

- Qualification of immersed gratings for Sentinel-5 passed. Flight models for both channels were produced and are now in integration and testing phase.

- Key optical parameters SWIR-3 IGs:

TIS $0.2 \%$

Efficiency $>58 \%$

Polarization sensitivity $<0.12$

WFE $<250 \mathrm{~nm}$ rms

- WFE dominated by thickness variations of wafer. Ion beam figuring or magnetorheological finishing for further reduction? Challenge is metrology of Si-wafers. 
SRON 
SRON 


\section{Additional slides}

\section{SRON}




\section{IG production - grating elements}

The target profile of the silicon grating elements has been determined with optical simulation in PCGrate.

\section{SWIR-3}

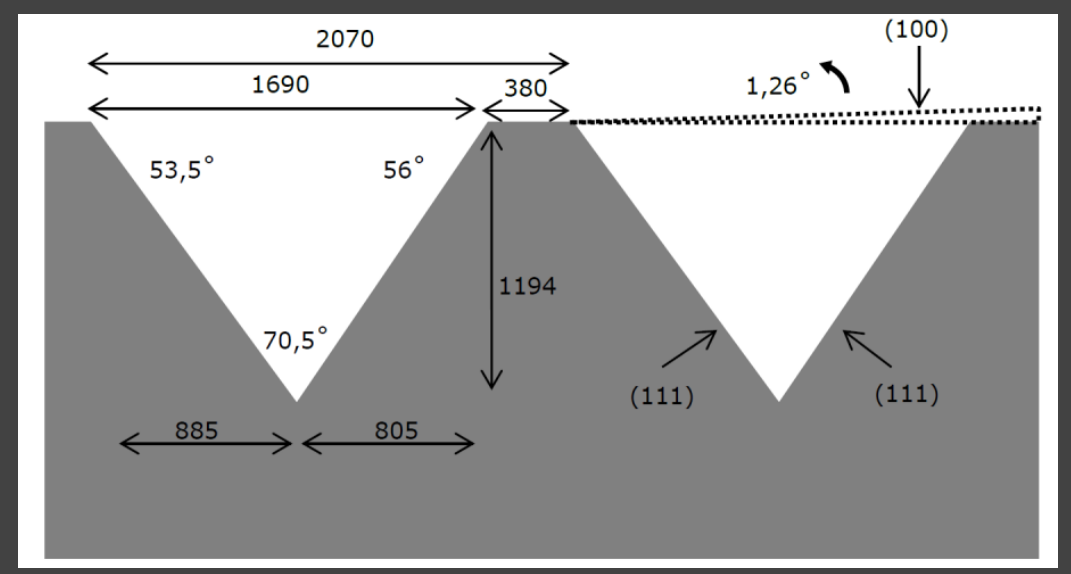

SWIR-1

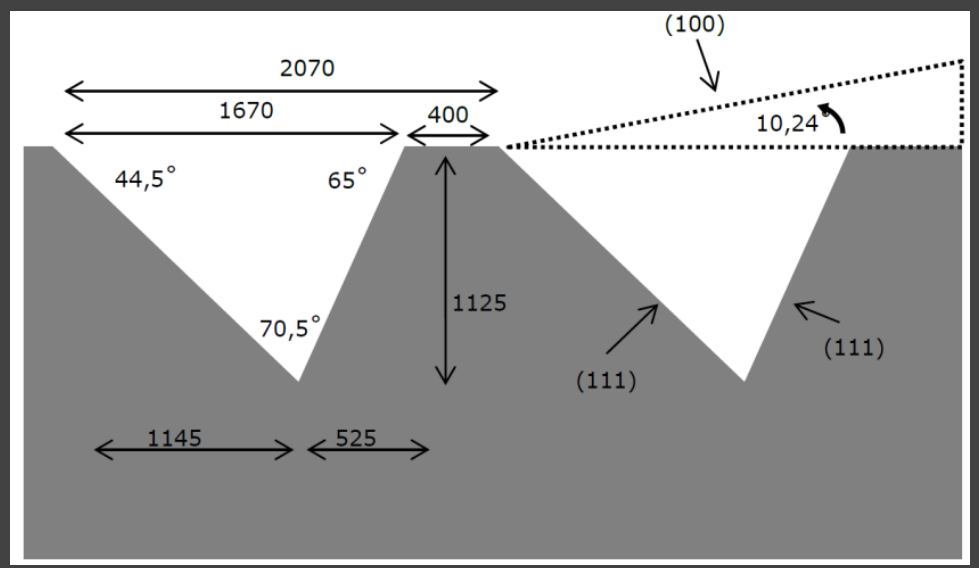

Production with UV lithography and anisotropic KOH etching for ultra-smooth surfaces

Angles are fixed by Si crystal structure and off-cut angle 


\section{IG production - bonding}

Perpendicularity verification with microscope

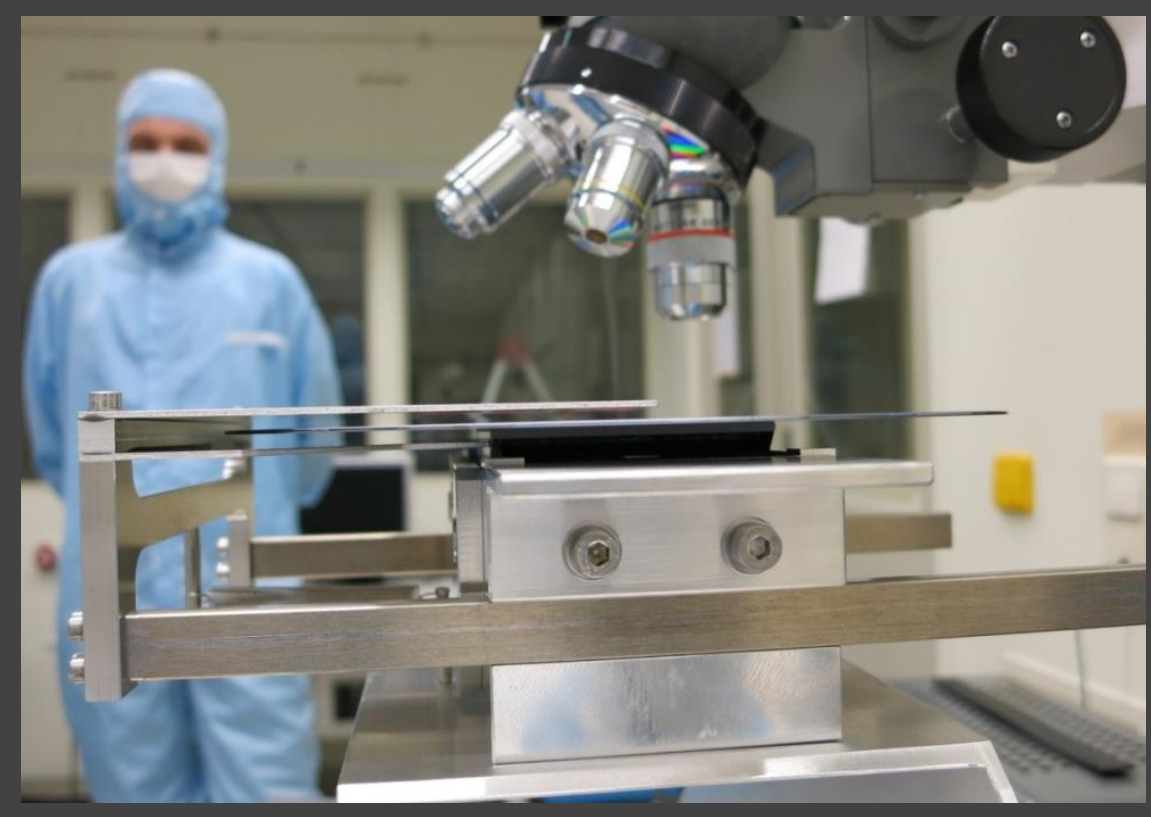

Image of setup under microscope

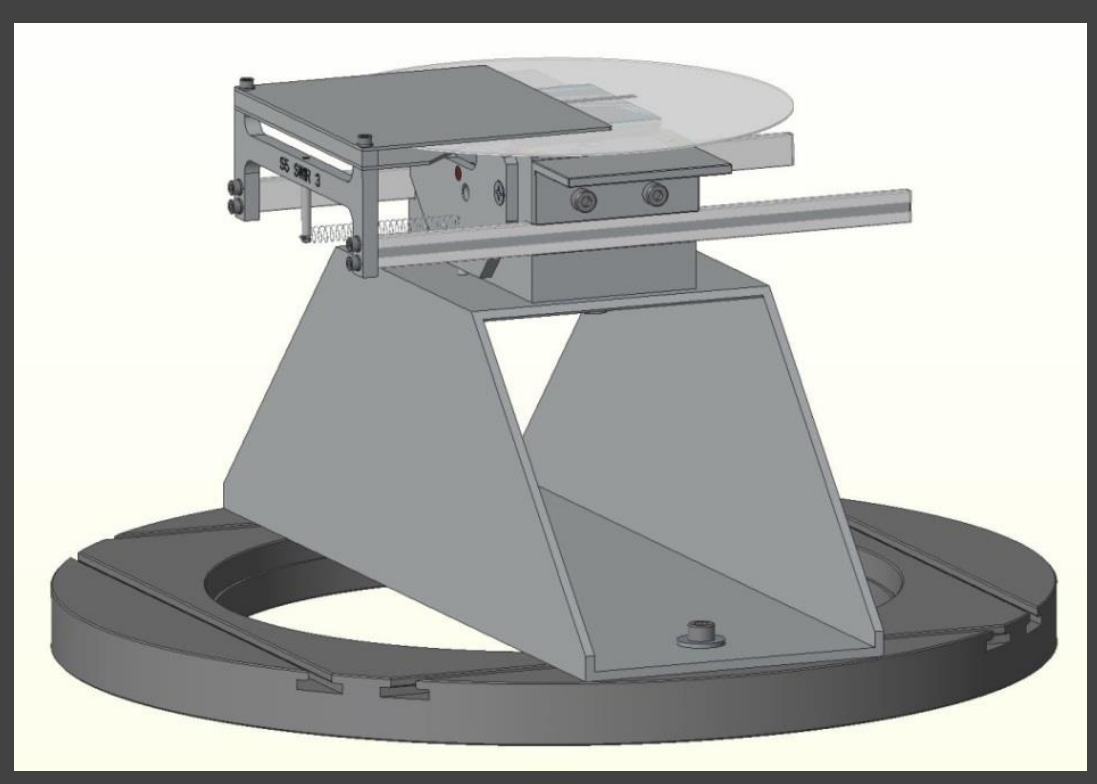

CAD drawing of measurement setup

Result: perpendicularity within $<100$ arcsec for all FMs

\section{SRON}


The Gratings need to be compliant with tight requirements on their geometry, see example of SWIR-3 IG drawing:
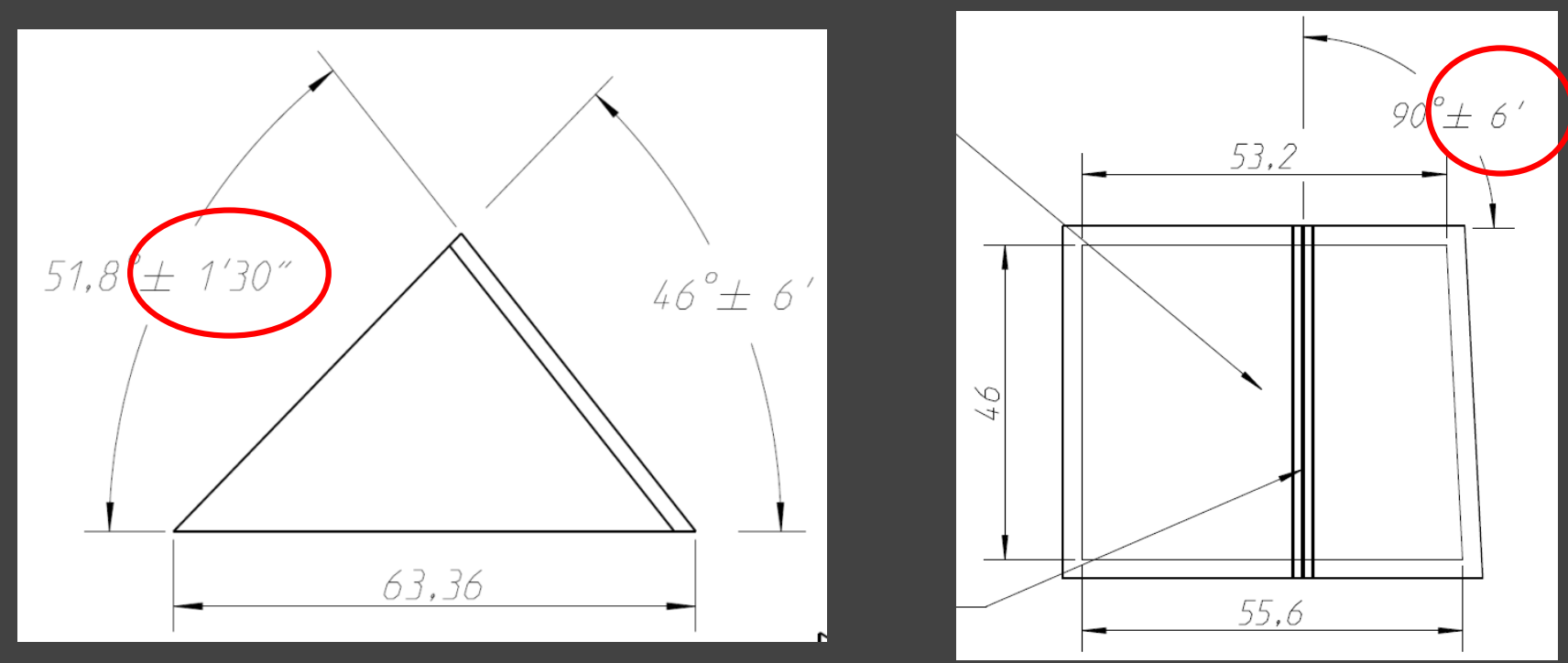

Further Grating lines periodicity of $(2070 \pm 4) \mathrm{nm}$ required IG rotation stability in housing has to be better than $0.2 \mathrm{arcsec} / \mathrm{K}$ 


\section{Grating Element Alignment Crosshairs}

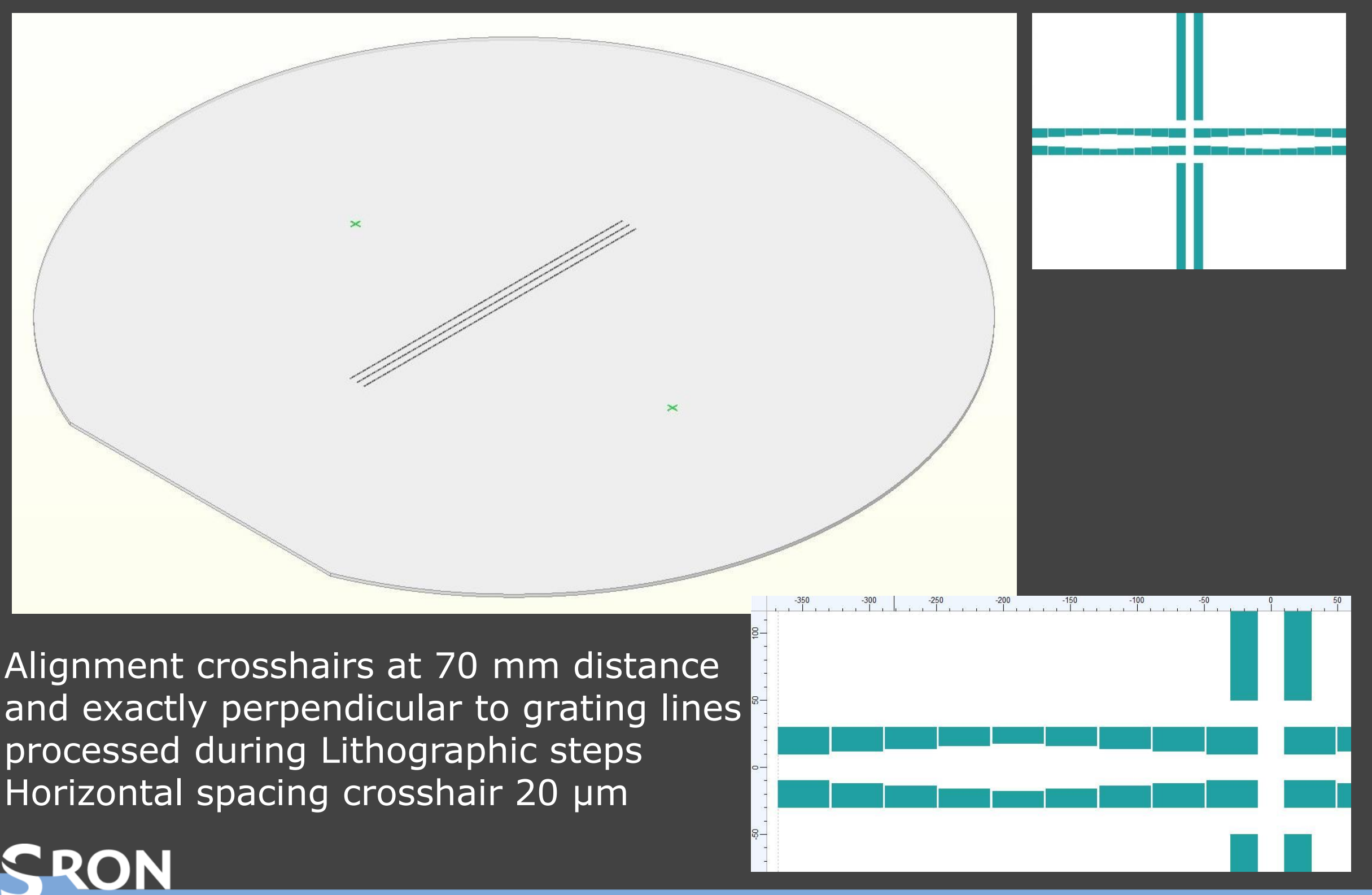


Alignment tool placed over prism and pressed to the reference side of prism by internal springs in support

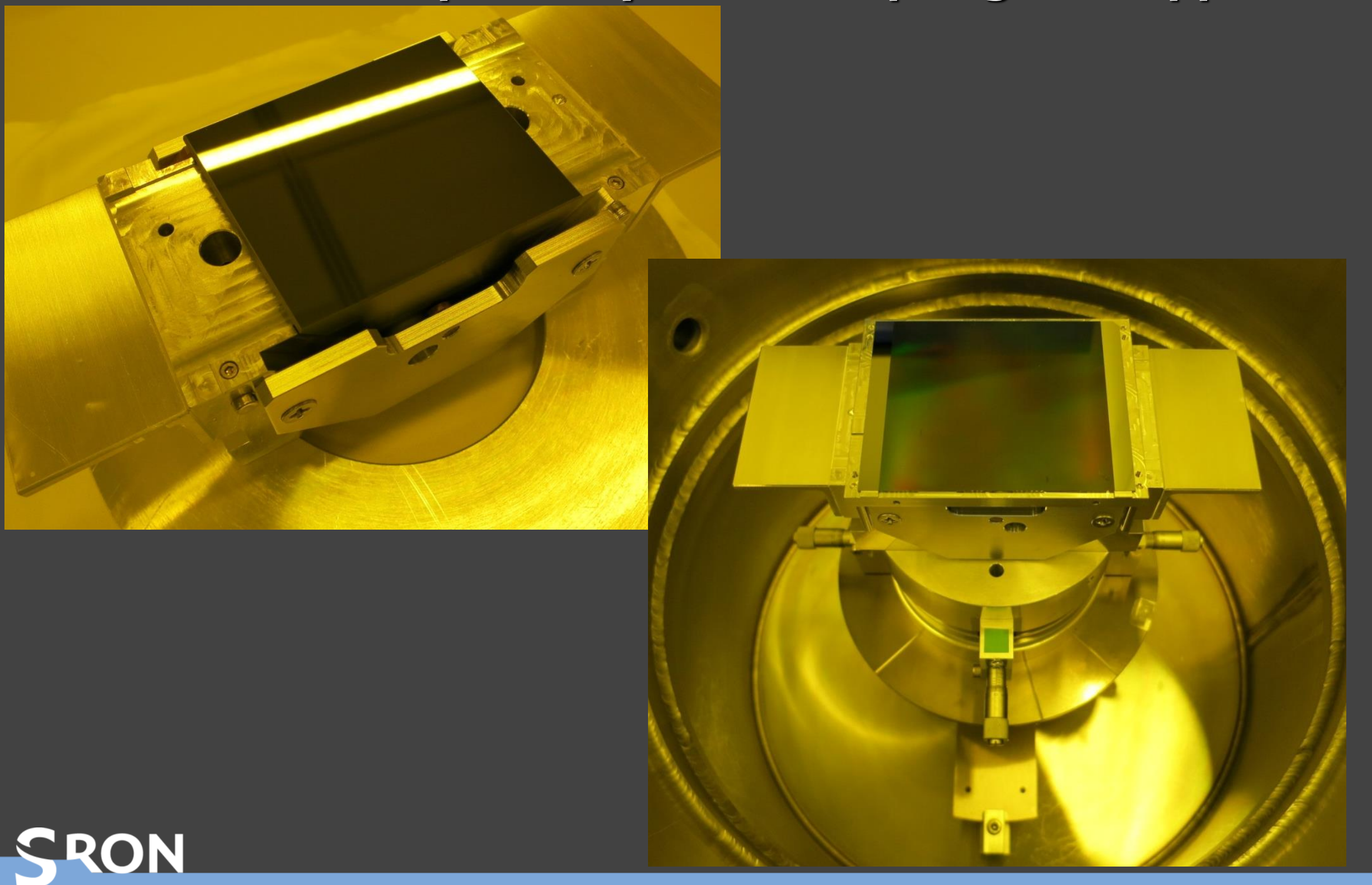




\section{Immersed Grating in the storage container}

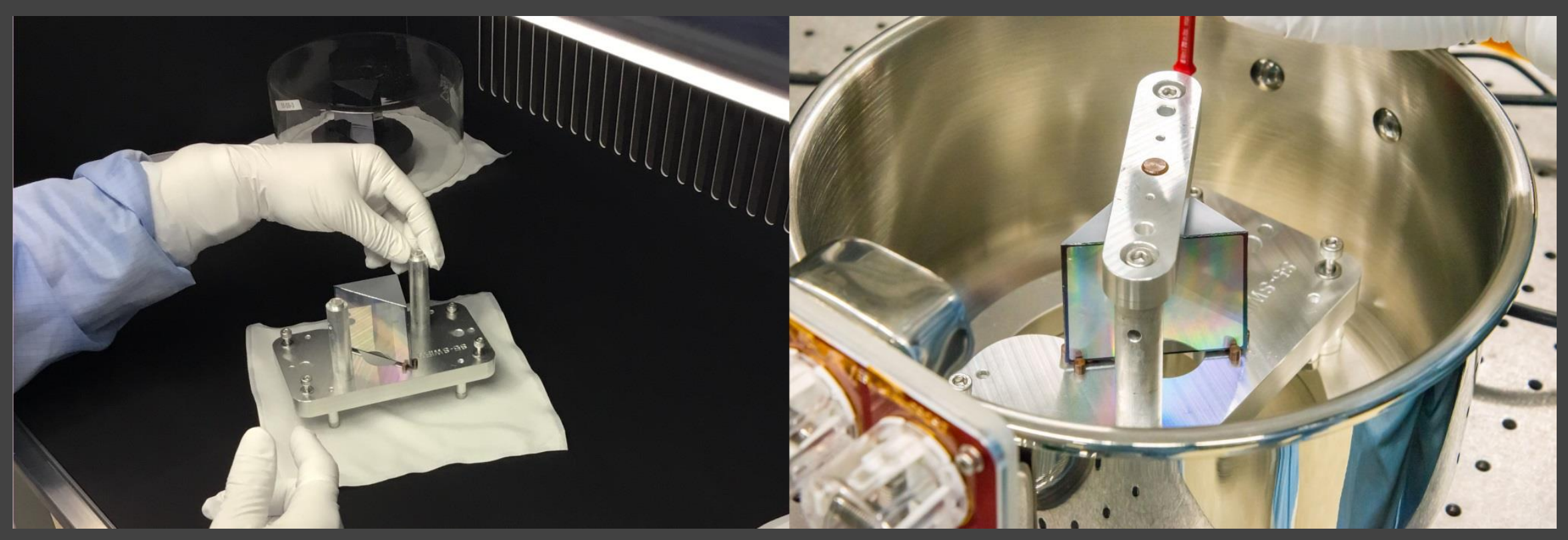




\section{IG production - grating elements}

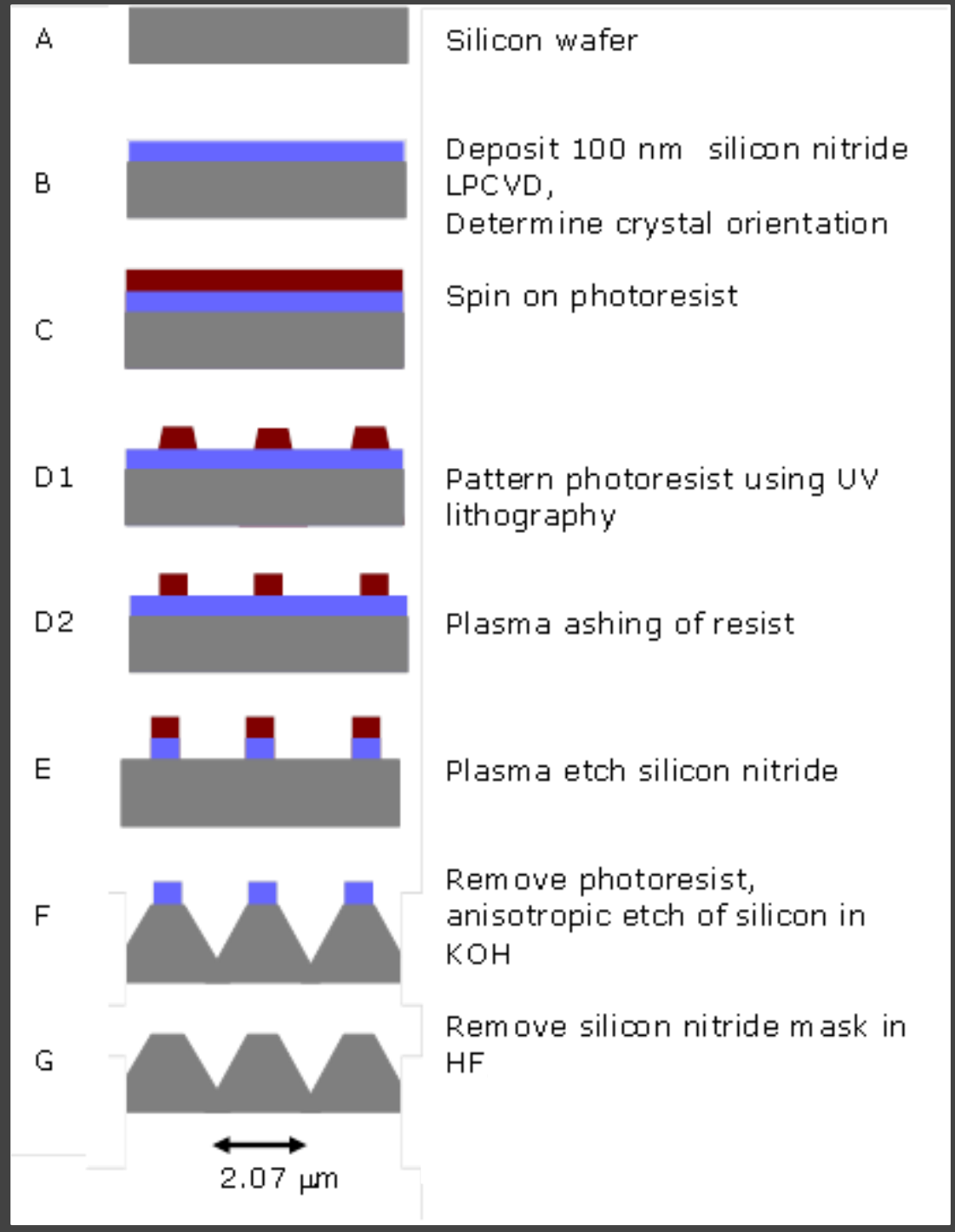

SRON
Standard UV lithography process

Start with float zone $150 \mathrm{~mm} \mathrm{Si}$ wafers

$500 \mathrm{~nm}$ linewidth on photomask leads to $380 \mathrm{~nm}$ dam width

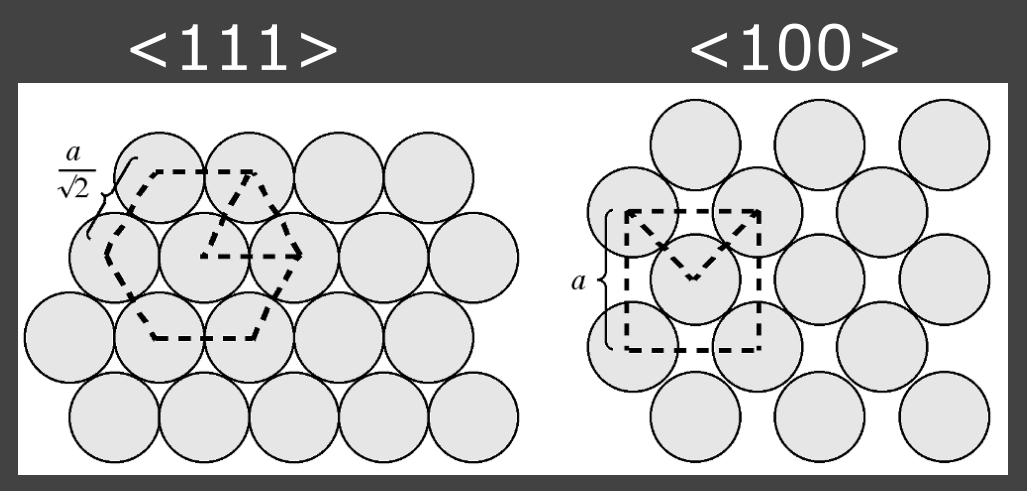

$\mathrm{KOH}$ two orders faster etching along $<100>$ than $<111>$ 